\title{
Les Phanariotes dans le roman roumain : un mythe littéraire
}

The Phanariots in Romanian Novels: a Literary Myth

\section{Andreia Roman}

\section{CpenEdition}

\section{Journals}

Édition électronique

URL : https://journals.openedition.org/ceb/4826

DOI : $10.4000 /$ ceb.4826

ISSN : 2261-4184

\section{Éditeur}

INALCO

Référence électronique

Andreia Roman, «Les Phanariotes dans le roman roumain : un mythe littéraire », Cahiers balkaniques

[En ligne], 42 | 2014, mis en ligne le 23 mai 2014, consulté le 07 juillet 2021. URL : http://

journals.openedition.org/ceb/4826; DOI : https://doi.org/10.4000/ceb.4826

Ce document a été généré automatiquement le 7 juillet 2021.

\section{c) (7) (5)}

Cahiers balkaniques est mis à disposition selon les termes de la Licence Creative Commons Attribution - Pas d'Utilisation Commerciale 4.0 International. 


\title{
Les Phanariotes dans le roman roumain : un mythe littéraire
}

The Phanariots in Romanian Novels: a Literary Myth

\author{
Andreia Roman
}

\section{Introduction}

1 Dans la littérature roumaine, le roman historique a privilégié, en lignes générales, une approche hagiographique de l'histoire nationale, insistant sur les épisodes de combat héroïque des Roumains pour l'indépendance ou pour la justice sociale. Cet aspect n'est pas spécifique de la littérature roumaine; né à l'époque romantique, comme support idéologique du "Printemps des peuples », il est présent dans toutes les littératures des Balkans et de l'Europe centrale. Néanmoins, ce filon thématique ne recouvre pas la totalité des sujets historiques. En Roumanie, dès les débuts du roman moderne et parallèlement à cette tendance idéalisante, nombre de romanciers se sont appliqués à mettre en scène des épisodes bien moins glorieux du passé national. Parmi ces derniers, l'époque des Phanariotes occupe une place de choix. Rapporté au regard des historiens sur la même époque, qui est complexe et nuancé, celui des romanciers est essentiellement négatif. Les Phanariotes sont perçus comme représentants d'un despotisme oriental qui a perverti les nobles traditions et valeurs ancestrales des Roumains, introduisant dans la vie politique et les mœurs quotidiennes la corruption, le clientélisme et le trafic d'influence, les mœurs dépravées, les intrigues, l'injustice sociale, le mépris du peuple, la spoliation des biens nationaux.

\section{Le double aspect des Phanariotes dans la littérature}

2 Ce type d'approche comporte deux aspects: le premier, d'ordre idéologique, conforte une vision traditionnellement victimiste, fréquente chez les Roumains, selon laquelle l'oppression étrangère qu'ils ont dû subir au cours de leur histoire a profondément entamé leur évolution économique, politique et surtout morale. L'autre aspect, d'ordre 
strictement littéraire, tient au côté exotique et pittoresque de cette époque, dont les tonalités fortes, riches en couleurs et en contrastes, constituent une indéniable source d'expressivité. Des épisodes tantôt épiques et pleins d'une violence dramatique, tantôt carnavalesques et truculents, s'offrent généreusement à leur imaginaire. Le pittoresque des personnages phanariotes tire son origine de l'ambivalence constitutive de leur statut: en tant que représentants de l'Empire ottoman, ils en adoptent la mentalité orientale, basée sur le despotisme, la corruption et la cruauté, en tant que Grecs, héritiers d'une ancienne et grande civilisation européenne, ils revendiquent leur appartenance à l'Europe chrétienne et aux valeurs culturelles de la modernité occidentale. Force est de rappeler, à ce propos, que la pénétration, dans les Principautés roumaines, de l'idéologie des Lumières est en bonne partie l'œuvre des Phanariotes. Au seuil du XIX $\mathrm{x}^{\mathrm{e}}$ siècle, les boyards roumains lisaient les œuvres de Voltaire, Montesquieu, Rousseau, Alfieri, etc. en langue grecque.

3 L'effet de contagion - qui affecterait l'ensemble de la société roumaine, au point d'en constituer une dimension «éternelle »- est l'une des idées récurrentes dans les romans inspirés de l'époque phanariote. Autrement dit, dans chaque roumain sommeillerait un phanariote prêt à exhiber ses tares dès que les circonstances historiques ou biographiques le permettent... Nous découvrons déjà le paradigme de cette idée dans Parvenus d'hier et d'aujourd'hui ${ }^{1}$ de Nicolae Filimon (1863), roman historique et social premier de la littérature roumaine moderne. L'auteur y présente l'ascension fulgurante de Dinu Păturică, jeune roumain de basse condition, qui fait ses premières armes en tant que serviteur à la cour d'un aristocrate phanariote. Doté d'une ambition sans bornes, Păturică parvient à ruiner son maître et à s'emparer de tous ses biens en se prévalant des mêmes méthodes jadis utilisées par celui-ci afin de parvenir à de hautes fonctions à la cour du prince grec Caragea.

Dans le roman de l'entre-deux-guerres, notamment chez Sadoveanu, Cezar Petrescu, Mateiu Caragiale, Hortensia Papadat-Bengescu, les références plus ou moins directes à l'héritage phanariote sont visibles, sans pour autant que le roman soit consacré entièrement à l'évocation de cette époque. Omniprésent dans la vie de la société moderne, cet héritage semble prédestiner celle-ci à un éternel immobilisme. Dans le roman Calea Victoriei, Cezar Petrescu énonce cette idée par la bouche d'Anton Muşat, vieux play-boy d'illustre ascendance : «Pour comprendre les gens et l'état des choses d'aujourd'hui, il faut remonter au temps des Princes Caragea et Moruz; c'est là qu'on trouve les racines, les coutumes et la tradition, tout ce qui est bon et ce qui est mauvais, ce qui nous a donné la vie et ce qui a causé notre déclin. L'occidentalisation n'est qu'une façade. Elle a changé le nom du pont de Mogoşoaia en Calea Victoriei [l'avenue de la Victoire] $\aleph^{2}$.

\section{Le revival des Phanariotes dans la littérature de l'époque Ceauşescu}

5 C'est en revanche à l'époque du communisme et notamment au temps de la dictature de Ceauşescu que l'évocation du temps des phanariotes connaît un véritable revival. Cette réactualisation est loin d'être anodine, ne serait-ce que par la volonté de certains écrivains de contrecarrer l'exhortation permanente des idéologues du régime à glorifier le passé héroïque du peuple roumain. Entre 1970 et 1987, nous retrouvons au moins quatre romans s'appliquant à consacrer entièrement leur thématique à ce sujet : 
Le Prince ${ }^{3}$ (1969) et La semaine des fous ${ }^{4}$ (1981) d'Eugen Barbu, Les faux-monnayeurs ${ }^{5}$ (1987) de Silviu Anghelescu et Privilèges ${ }^{6}$ (1987) de Maria Luiza Cristescu. L'effet de contamination que nous venons d'évoquer est parfaitement décrit dans le dernier roman cité. Paraphrasant la célèbre formule «Byzance après Byzance » utilisée par Nicolae Iorga en référence au Moyen âge roumain, on peut intituler ce roman comme «Le phanariotisme après les Phanariotes ». L'action du roman a lieu en 1837, à la cour $\mathrm{du}$ prince moldave Mihail Sturza, un aristocrate aux lointaines ascendances grecques qui, tout en se proclamant Roumain, ne renonce à aucun des abus pratiqués par ses ancêtres. Les mœurs de la cour en sont à tel point imprégnées qu'elles parviennent à contaminer même les esprits les plus éclairés et les plus attachés aux idéaux de la modernité occidentale. C'est le cas du protagoniste, un jeune intellectuel français, nourri d'idées libérales et révolutionnaires, qui, pour fuir le régime de la Restauration instauré en France, se réfugie à la cour du hospodar moldave, devenant le précepteur de ses fils. Or, au cours de son périple roumain, ce Français constate tout d'abord que l'héritage phanariote est bien vivant et, au lieu de parvenir à le contrecarrer par les valeurs occidentales dont il est porteur, il finit par en adopter lui même les pratiques et s'en approprier l'arrivisme, l'hypocrisie et le cynisme. Bref, il devient plus phanariote que les Phanariotes.

6 Les faux monnayeurs abordent l'époque phanariote par son versant plébéien. Le héros du roman est le prince Nicolae Mavrogheni, ancien pirate grec, un rustre aventurier qui a régné en Valachie entre 1786 et 1790 . L'auteur exploite le côté grivois, cocasse et grotesque de l'époque évoquée dont le protagoniste, décrit comme un homme inculte, brutal, cynique et surtout mégalomane, présente une ressemblance frappante avec le président Nicolae Ceauşescu. Cette ressemblance à peine voilée a fait à l'époque les délices des lecteurs, dont le sport national consistait justement à détecter dans la littérature les moindres références allusives à la dictature et qui ont accueilli le roman avec enthousiasme, mettant la censure dans une posture plus qu'embarrassante. Comment fallait-il réagir? Interdire le roman équivalait à reconnaître le bien-fondé de ces allusions satiriques. La censure a donc préféré adopter l'alibi dont l'auteur luimême s'était prévalu : Mavrogheni était un Grec, mandataire d'un pouvoir oppresseur étranger; à l'abri, donc, de tout soupçon d'hérésie, le choix thématique de l'auteur caressait dans le sens du poil le national-communisme exalté par le Conducător.

7 C'est le même alibi dont se prévaut Eugen Barbu dans ses deux romans inspirés de la même époque phanariote, évoquant cette fois-ci son moment crépusculaire. L'auteur met en scène l'atmosphère de décadence, de fin de règne précédant l'insurrection valaque de 1821 qui a mis fin à cette domination. Le héros du premier roman est un prince dont le nom n'est pas indiqué; il règne despotiquement dans une Valachie dévastée par la peste et par les exactions sauvages infligées à la population, et finit par être décapité sur l'ordre du Sultan. Le personnage est profondément ambivalent : sa soif de pouvoir, sa sensualité délirante et perverse sont doublées en permanence d'un mal de vivre, d'un sentiment de l'aléatoire et de présages d'une fin imminente. Le Prince est à la fois un personnage odieux et tragique. Le héros de La semaine des fous est un boyard phanariote, Hrisanti Hrisoscèl, membre de la société secrète de l'Hétairie qui prépare l'insurrection grecque anti-ottomane. En tant que trésorier de cette société, il dépense l'argent qui lui est confié dans une vie de débauche menée à Venise en compagnie d'une belle courtisane vénitienne. Les deux personnages - le Prince et Hrisanti Hrisoscel - sont, à l'opposé de N. Mavrogheni, le héros de Silviu Anghelescu, 
des spécimens d'aristocrates raffinés, nourris de culture occidentale, poussant leur raffinement aux extrêmes limites de la perversité et de la décadence ; des êtres à la fois blasés et cyniques. Ils se présentent donc comme le produit fatal d'un mal historique dont ils sont à la fois responsables et victimes. Or, il y a dans ces deux romans une certaine implication subjective qui nous fait penser à une possible projection autobiographique de l'auteur. En termes flaubertiens, cela reviendrait à dire « Le Prince c'est moi ! », « Hrisanti Hrisoscel, c'est encore moi ! » Pour cynique qu'il paraisse, un tel aveu s'avère fort plausible. La parution posthume d'un roman autobiographique - voire un roman-testament - portant le titre significatif Janus lève en partie le voile sur la personnalité controversée du romancier. Incarné par le personnage mythologique, divinité à double face, l'auteur évoque et justifie sa duplicité - son comportement de victime et de coupable à la fois - à l'époque du communisme. La couverture du livre présente d'ailleurs son portrait en forme de tête à double face, l'une souriante, l'autre sombre et menaçante. À l'image de son héros fictif, le Prince blasé et sans scrupules, E. Barbu se présente lui-même comme le produit fatal d'un système politique qui réactualise mutatis mutandis les pratiques phanariotes sous tous leurs aspects et dont il contemple avec une sorte de joie ricaneuse le spectacle grotesque. Voici ce qu'il raconte à l'occasion d'un rassemblement festif du Comité central dont il est l'invité d'honneur : « je me sentais toujours, dans des occasions pareilles, comme dans une Byzance fictive, lorsque, entouré des drapeaux et au son des graves hymnes grecs, je me sentais transporté avec une sorte de volupté par l'ivresse de ces masses dont le bel et sincère enthousiasme avait été préparé en détail et pendant des journées entières par des animateurs bien instruits $»^{7}$.

\section{Conclusion}

8 Issu d'une réalité historique complexe, qui ne cesse de dévoiler aux historiens ses multiples faces, le temps des Phanariotes s'est constitué, dans l'imaginaire des écrivains roumains, en un véritable mythe littéraire. Les fictions romanesques que nous venons de présenter montrent qu'au fil du temps et des différentes étapes du passé national, ce mythe fut fortement instrumentalisé, étant destiné à livrer un message politique ou idéologique par excellence réprobateur. La voie d'une approche plus objective - voire plus nuancée - capable de restituer cette époque dans l'intégralité de ses zones d'ombre et de lumière est demeurée jusqu'à présent inexplorée.

\section{BIBLIOGRAPHIE}

Anghelescu Silviu, (1987), Calpuzanii, Bucarest : Cartea românească.

Barbu Eugen, (1969), Principele, Bucarest : Editura Tineretului.

Barbu Eugen, (1981), Săptămâna nebunilor, Bucarest : Albatros.

Barbu, Eugen (1993), Ianus, Bucarest : Ed. Gramar. 
Cristescu Maria Luiza, (1987), Privilegiu, Bucarest : Cartea românească.

Filimon Nicolae, (1863), Ciocoii vechi şi noi, Bucarest.

Petrescu Cezar, (1985), Calea Victoriei, Bucarest : Cartea românească.

\section{NOTES}

1. Nicolae Filimon, Ciocoii vechi şi noi, Bucureşti, 1863.

2. Cezar Petrescu, Calea Victoriei, Cartea românească, Bucarest, 1985, p. 77.

3. Eugen Barbu, Principele, Editura Tineretului, Bucarest, 1969.

4. Eugen Barbu, Săptămâna nebunilor, Albatros, Bucarest, 1981.

5. Silviu Anghelescu, Calpuzanii, Cartea românească, Bucarest, 1987.

6. Maria Luiza Cristescu, Privilegiu, Cartea românească, Bucarest, 1987.

7. E. Barbu, Ianus, Éd. Gramar, Bucarest, 1993, p 16.

\section{RÉSUMÉS}

Dans la littérature roumaine, le temps des Phanariotes a représenté une incessante source d'inspiration. Ce thème prend son essor dans le premier roman moderne, Parvenus d'hier et d'aujourd'hui de Nicolae Filimon (1863), et connaît un véritable revival à l'époque du communisme à travers les romans de Maria Luiza Cristescu, Silviu Angelescu et Eugen Barbu. À la différence des historiens dont le regard sur l'époque phanariote s'applique à être objectif et nuancé, les romanciers, privilégiant une approche partiale, ont porté, au fil des différentes étapes historiques, un jugement essentiellement négatif sur cette période. Selon les auteurs cités, le régime phanariote a instauré dans la société roumaine l'esprit de corruption, l'arbitraire des décisions, le règne des privilèges, le népotisme et la dépravation. Le phénomène phanariote, loin d'être présenté comme un accident de l'histoire, apparaît ainsi comme une dimension constitutive, voire « éternelle » de l'ontologie roumaine.

In Romanian literature, the Phanariote era has been an unending source of inspiration. The theme first appeared in the first modern novel, The old and the New Parvenus by Nicolae Filimon (1863), and experienced a true revival during the Communist era through the novels of Marial Luiza Cristescu, Silviu Angelescu and Eugen Barbu. Unlike historians whose stance on the Phanariote era attempts to be objective and nuanced, novelists, preferring a partial approach, have essentially looked negatively on that period throughout history. According to the aforementioned authors, the Phanariote regime instilled in Romanian society a spirit of corruption, arbitrary decisions and the reign of privileges, nepotism and depravation. The Phanariote phenomenon, far from being presented as an accident of history, thus appears as a constitutive, sometimes "eternal" dimension of Romanian ontology. 
INDEX

motsclestr Fenerliler, Romanya, On dokuzuncu yüzyıllarda, Yirminci yüzyıllarda, Edebiyat ve Tarih, Edebiyat, Romen Edebiyat

Keywords : Phanariots, Romania, Nineteenth century, Twentieth century, History and literature, Literature, Romanian literature

Thèmes : Littérature

motsclesmk ФАНАР, РОМАНИЈА, ДЕВЕТНАЕСЕТТИОТ ВЕК, ДВАЕСЕТТИОТ ВЕК, ЛИТЕРАТУРА ОД ИСТОРИЈА, ЛИТЕРАТУРА, РОМАНСКАТА ЛИТЕРАТУРА

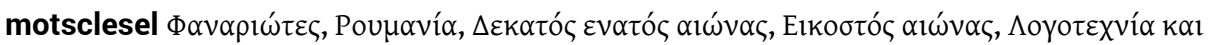

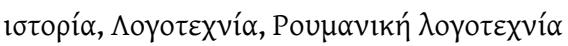

Index géographique : Roumanie

Mots-clés : Phanariotes, Filimon Nicolae (1819-1865), Petrescu Cezar (1892-1961), Barbu Eugen

(1924-1993), Anghelescu Silviu (1925-), Cristescu Maria Luiza (1943-2001)

Index chronologique : dix-neuvième siècle, vingtième siècle

\section{AUTEUR}

ANDREIA ROMAN

$\mathrm{MCF}$

INALCO 